OAI-PMH: http://www.indteca.com/ojs/index.php/Revista Scientific/oai

Artículo Original / Original Article

\title{
La Reforestación como Estrategia Ambiental para la Conservación de ríos y quebradas
}

Autor: Yely Alexander Molina Pereira Universidad Pedagógica Experimental Libertador, UPEL molinaalex1605@gmail.com

Barinas, Venezuela https://orcid.org/0000-0003-3876-3962

Resumen

La investigación tiene como objetivo Implementar la Reforestación como Estrategia Ambiental para la Conservación de ríos y quebradas; precisando la realidad mediante una investigación de naturaleza cuantitativa, apoyada en la modalidad de Proyecto Factible y sustentada en un diseño de campo, cumpliendo con las fases de: diagnóstico, factibilidad, diseño, ejecución y evaluación. La población la conformaron treinta personas, tomando como muestra su totalidad. La técnica empleada fue la encuesta y el instrumento un cuestionario tipo escala de Likert con cinco alternativas de respuestas, validado por expertos y establecida su confiabilidad a través del coeficiente de Alpha de Cronbach, siendo analizados e interpretados mediante gráficos de barras. Con el cambio climático, se hace evidente la necesidad de reforestar las fuentes hídricas garantes del abastecimiento de agua de animales, cultivos y en el caso particular de la Presa Manuel Palacio Fajardo. La falta de cuidado de este recurso natural, justificó la adopción de estrategias para mitigar el daño ambiental y promover el cuidado. Pudiéndose plantar doscientos cincuenta árboles de Bambú gracias al apoyo y colaboración de los habitantes al participar en la reforestación.

Palabras clave: reforestación; conservación ambiental; hidrografía; medio ambiente.

Fecha de Recepción: 22-02-2019
Fecha de Aceptación: 30-05-2019
Fecha de Publicación: 05-08-2019 


\title{
Reforestation as Environmental Strategy for the Conservation of rivers and streams
}

\begin{abstract}
The objective of the research is to Implement Reforestation as Environmental Strategy for the Conservation of rivers and streams; specifying the reality by means of a quantitative research, supported by the Feasible Project modality and supported by a field design, complying with the phases of: diagnosis, feasibility, design, execution and evaluation. The population was made up of thirty people, taking as a sample its totality. The technique used was the survey and the instrument a Likert scale questionnaire with five alternative answers, validated by experts and established its reliability through the Cronbach's Alpha coefficient, being analyzed and interpreted by bar graphs. With climate change, it becomes evident the need to reforest the water sources that guarantee the water supply of animals, crops and, in the particular case of the Manuel Palacio Fajardo Dam. The lack of care for this natural resource justified the adoption of strategies to mitigate environmental damage and promote care. Being able to plant two hundred and fifty Bamboo trees thanks to the support and collaboration of the inhabitants when participating in the reforestation.
\end{abstract}

Keywords: reforestation; environmental conservation; hydrography; environment.

Date Received: 22-02-2019
Date Acceptance: 30-05-2019
Date Publication:

05-08-2019 


\section{Introducción}

La reforestación es necesaria para mantener en buen estado el ambiente natural y los recursos que son esénciales e importantes para la vida, entre los cuales se pueden mencionar: el agua, el aire y el suelo. Además, dicha labor contribuye en brindar protección a otros elementos; por lo tanto, se debe aplicar en las áreas cercanas a las cuencas existentes y áreas protegidas como los parques nacionales. También se localizan sitios que requieren protección arbórea tales como: las orillas de los ríos, los ojos de agua, sitios de recarga acuífera y las microcuencas que abastecen las tomas de agua de los acueductos rurales, sitios con pendientes muy fuertes que no permiten desarrollar otras actividades.

En tal sentido, se deben aplicar estrategias para la reforestación de diversas especies de árboles que favorezcan la conservación de los bosques, así como también motivar a los habitantes de las comunidades para que participen activamente en los proyectos ambientales; con este enfoque, el investigador promueve la siembra de bambú "Guadua angustifolia Kunth" con la finalidad de Implementar la Reforestación como Estrategia Ambiental para la conservación de ríos y quebradas, realizando para ello, un diagnóstico sobre las especies forestales apropiadas para la forestación de la zona, determinando la factibilidad y a su vez diseñando y aplicando estrategias ambientales para motivar a los habitantes del sector a participar activamente en la reforestación.

Es oportuno señalar que, el daño ambiental y la deficiencia del recurso hídrico causado por fenómenos naturales y la falta de cuidado de los mismos, justificó la adopción de estrategias para controlar, enmendar y promover el cuidado de ríos y quebradas; aunque existen entidades que están encargadas de velar por el cuidado de los recursos naturales, hasta la fecha los pobladores no han contado con el apoyo para la protección de la zona; por ende, se orientó a los habitantes en cuanto al resguardo de los bosques y sean ellos los que 
realicen proyectos ambientales en la comunidad proporcionando el cuidado y mantenimiento a los recursos naturales, garantizando asimismo, su derecho humano de vivir en un ambiente libre de contaminación.

En tal sentido, el Ministerio del Poder Popular para la Educación (MPPE) debe implementar estrategias innovadoras que se complementen con la educación ambiental tomando en cuenta los valores, motivando a los estudiantes, profesores y comunidad en general a participar en el proceso educativo para contribuir con el bienestar de las familias y las comunidades, en aras de fortalecer la educación ambiental, ya que esta es relativamente nueva, la razón es que el ser humano antes contaminaba menos su entorno o quizás no lo hacía; fue con el inicio de la industrialización y el gran aumento de la población mundial en el planeta, como empezó a disminuir la calidad de nuestro entorno y en consecuencia se hace evidente el deterioro ambiental.

Con referencia a lo anterior, González (2016), en su trabajo de investigación denominado: "Proyecto de reforestación de la quebrada La Pilama, mediante la implementación de dos viveros para el abastecimiento de material vegetal en la vereda Liberia (Viotá Cundinamarca)" (pág. 1); en la Universidad de Cundinamarca, cuyo objetivo fue establecer dos viveros de propagación y producción de material vegetal para la reforestación y recuperación de la quebrada la Pilama, garantizando el acceso continuo del recurso hídrico de la población de la vereda Liberia del Municipio de Viotá. El propósito del proyecto fue integrar actividades desde la producción de material vegetal hasta la reforestación.

Por su parte, Lara (2017), en su trabajo de investigación titulado: "Determinación de cambios de distribución de especies por efectos del cambio climático en el parque municipal natural Campo Alegre y parque regional natural Ucumarí en Risaralda Colombia" (pág. 1). Las áreas protegidas pueden ser vulnerables al cambio climático, problemática que ha hecho que se concentren la mayor parte de los esfuerzos para su protección a nivel mundial. 
Con el propósito de determinar si los bosques del Parque municipal natural de Campo Alegre y el Parque regional natural de Ucumarí en Risaralda Colombia, por lo que en la presente investigación se ha seleccionado una especie de planta (Bambú) a fin de, contribuir con el aumento de los espacios boscosos de la localidad.

En tal sentido el objetivo de la investigación es Implementar la Reforestación como Estrategia Ambiental para la conservación de ríos y quebradas, contribuyendo de esta manera con el cuido y resguardo del ambiente.

\section{Metodología}

La investigación se enmarca en la modalidad de proyecto factible cuyo propósito fue la reforestación como estrategia ambiental en la conservación del caudal hídrico de ríos y quebradas. De acuerdo con, el Manual de Trabajos de Grado de Especialización y Maestría y Tesis Doctorales de la Universidad Pedagógica Experimental Libertador (UPEL, 2016a), expresa que un proyecto factible: "consiste en la investigación, elaboración y desarrollo de una propuesta de un modelo operativo viable para solucionar problemas, requerimientos, o necesidades, de organizaciones o grupos sociales; puede referirse a la formulación de políticas, programas, tecnologías, métodos o procesos..." (pág. 21).

Por consiguiente, el propósito de la investigación se centró en dar respuesta a las necesidades de un colectivo y del ambiente en general con el fin de mitigar y en lo posible revertir los daños ocasionados al ambiente a causa de las malas decisiones de los anteriores pobladores de la comunidad.

\subsection{Diseño de la investigación}

El estudio fue abordado considerando la investigación de campo, que de acuerdo con el Manual de Trabajos de Grado de Especialización y Maestría 
y Tesis Doctorales de la UPEL (2016b):

Es el análisis sistemático de problemas en la realidad, con el propósito bien sea de describirlos, interpretarlos, entender su naturaleza y factores constituyentes, explicar sus causas y efectos, o predecir su ocurrencia, haciendo uso de métodos característicos de cualquiera de los paradigmas o enfoques de investigación... (pág. 18).

Con base en la anterior definición, el estudio abordó la problemática, partiendo de un diagnóstico que permitió comprender la naturaleza del problema, sus causas y consecuencias, para posteriormente analizar y reflexionar la realidad existente, es importante agregar, que la investigación de campo se basa en: descripciones, criticas, reflexiones, interpretación, análisis, evaluación y a su vez se recoge la información directamente de su fuente, apoyándose eventualmente en la revisión de material bibliográfico, como soporte o fundamento a la investigación.

\subsection{Reforestación}

Según Hernández (2010), la reforestación es definida como: "un conjunto de actividades que comprende la planeación, la operación, el control y la supervisión de todos los procesos involucrados en la plantación de árboles" (pág. 11). Partiendo de la anterior definición, la reforestación es una práctica ecológica-ambiental donde se lleva a cabo las múltiples actividades planificadas en aras de sembrar cualquier variedad de plantas, con el fin de poblar espacios de terreno que le ha sido arrebatada la capa boscosa por las causas que haya sido, haciendo del espacio el lugar propicio para el resguardo de especies de fauna de las zonas cercanas, y a su vez contribuir positivamente con la conservación de la fuentes hídricas del territorio. 
Por su parte, Mendoza (2017), en su trabajo de investigación plantea que:

La reforestación hace relación a la siembra de árboles en terrenos con aptitud forestal que en el pasado albergaron bosques los cuales desaparecieron por diversos motivos y es altamente beneficiosa por los bienes que se producen y los servicios ambientales que presta (pág. 16).

Con relación, a la anterior definición se asume a la reforestación como una alternativa para revertir los daños causados al ambiente, a fin de devolverle a la naturaleza parte de su estado natural, que con el paso del tiempo el hombre por desconocimiento, para mejorar su poder adquisitivo entre otras cosas, ha venido deteriorando. Es oportuno indicar, que la actividad de reforestación se realizó durante el periodo de lluvia del año 2018, a fin de evitar la pérdida del material vegetal a causa del periodo seco.

\subsection{Estrategia Ambiental}

Una estrategia ambiental puede ser considerada como el resultado de un proceso de análisis, en el que se han de formular e implementar diferentes medidas de protección ambiental, y que estas a su vez persiguen ciertos objetivos, en función de mitigar o revertir los daños causados al ecosistema. En tal sentido, Rodríguez (s.f.), afirma que:

El termino Estrategias Ambiéntales es mencionado por primera vez en la Agenda 21 documento que fue suscripto en la Cumbre de la Tierra, la más vasta reunión de dirigentes mundiales, que se celebró en (el mes de junio) de 1992, en Río de Janeiro (Brasil). En donde se exhorta a los gobiernos a que adopten estrategias nacionales para el desarrollo sostenible. Estas deberán elaborarse con la amplia participación de todos los sectores, incluidas las organizaciones no gubernamentales y el público en general (pág. 2).

Por las consideraciones anteriores, una estrategia ambiental impulsa 
retos y oportunidades, con el objeto de generar y mejorar de manera permanente los espacios y que a su vez garanticen a la colectividad una gestión ambiental responsable, considerando este proceso como un conjunto de medidas, que involucra a todo el personal, orientándolo a disminuir los daños ambientales, así como promover la concienciación en cuanto uso adecuado de los recursos naturales y en consecuencia del ambiente como un todo, necesario e indispensable para la supervivencia de la vida en el planeta.

\subsection{Conservación}

La acepción más extendidamente aceptada fue presentada por la Unión Internacional para la Conservación de la Naturaleza y Recursos Naturales (UICN, 1980), citado por Hernández (2011), definiéndola como: "La utilización humana de la biosfera para que rinda el máximo beneficio sostenible, a la vez que mantiene el potencial necesario para las aspiraciones de futuras generaciones" (pág. 14); en concordancia con la definición anterior, la conservación puede ser entendida como el uso consciente y racional de recursos que nos provee la naturaleza asegurando en consecuencia, la permanencia de la vida de todas las especies en el planeta, sin alterar su espacio y condiciones naturales, propiciando un ambiente agradable y ameno para la existencia y permanencia de la vida en planeta tierra.

De la misma manera, se tiene que, conservar no es más que evitar la sobre explotación de los recursos que nos provee la naturaleza, asegurando a las futuras generaciones un espacio garante de la vida y por ende de la existencia, es adquirir responsabilidad en nuestro quehacer y el compromiso de la sociedad de fomentar razonablemente el equilibrio ambiental de la presente y las venideras generaciones.

\subsection{Conservación Ambiental}

En la actualidad se cuenta con autores quienes han dado sus aportes 
teóricos en función a la conservación y al ambiente, sin embargo se observa bajo investigaciones que hasta la fecha no se encuentra un constructo exacto respeto a esta definición por lo que se propone como conservación ambiental al uso consciente de los recursos que nos oferta la naturaleza, garantizando a las futuras generaciones un ambiente sanamente aceptable, reconociendo y mejorando la permanencia de los ecosistemas, así como de la calidad de vida de la población.

En relación a lo expuesto y en consideración a la investigación, la propuesta definida se apoyó en la clara evidencia y degradación que se ha observado a los alrededores de ríos y quebradas, donde se desarrolló la reforestación en aras de contribuir con la conservación del ambiente, y en efecto se realizó la plantación de doscientos cincuenta (250) plantas de bambú "Guadua angustifolia Kunth"

\subsection{El Problema}

El deterioro del planeta y en consecuencia del ambiente en general tiene varios orígenes o causas y todo depende del nivel cultural y social del individuo, de su compromiso y del respeto de este por el ambiente, la falta de conciencia y la irresponsabilidad de los entes gubernamentales, trae como consecuencia la perdida de la biodiversidad causada por la destrucción del hábitat, contaminación y sobre explotación; el estado venezolano desde hace más tres (3) lustros adelanta evaluaciones de los problemas ambientales, así como la gestión y estrategias de solución, ya que se considera que la forestación contribuye positivamente con la conservación y recuperación ambiental, en la producción de oxígeno, elemento indispensable para la existencia de la población en el planeta.

Por su parte, Rojas (2007a), en su investigación hace mención, a tres modelos de regulación ambiental: Mercado, Estado y Comunidad; quien expresa: 
El papel del Estado como administrador directo de los recursos no escapa a las críticas, pues raramente ejerce eficientemente sus facultades regulatorias en las tierras del dominio público y privado de la nación. Cuando decreta áreas especiales con fines protectores (parques nacionales, reservas de biosfera) 0 con fines productores (reservas forestales, zonas agrícolas) por lo general carece de los mecanismos institucionales y financieros para aplicar social y ecológicamente las medidas de regulación. En consecuencia, se producen conflictos de objetivos e intereses con los pobladores y productores de los territorios, debido al poco espacio de participación que se les reserva a los usuarios en la formulación de las normativas ambientales y territoriales (pág. 132).

En tal sentido, se puede evidenciar el deterioro como consecuencia de la tala y la quema de gran cantidad de hectáreas de bosque y la administración nacional se ha desentendido ante esta grave situación, por lo que se puede decir, que sin el apoyo de personas e instituciones comprometidas con el accionar ambiental, donde su fin esencial sea la conservación y resguardo de los espacios naturales, dejando de lado los intereses particulares, para de esta manera evitar la destrucción de la naturaleza a causa de la explotación desmedida e inconsciente de sus recursos, con el propósito de lograr reorientar la gestión pública a fin de mitigar el deterioro ambiental.

Por su parte, el estado Barinas cuenta con organizaciones como: el Ministerio del Ambiente, MINEA, INPARQUES y algunos espacios naturales, como: el Parque Moromoy, Barinitas, el Parque la Federación, Jardín Botánico de la Universidad Nacional Experimental de Los Llanos Occidentales Ezequiel Zamora (UNELLEZ), Barinas, Parque Natural la Ciénega, Barrancas, en apoyo y resguardo de las zonas que son considerados pulmones vegetales del estado. Sin embargo, se observan acciones que han atentado contra las especies forestales, y por ende el deterioro y la degradación del ambiente.

En tal sentido, las áreas boscosas han de ser valoradas en respuesta a la variedad de beneficios que estas ofrecen a la sociedad en general, siendo 
oportuno indicar, que la plantación de árboles y resguardo de los bosques es fundamental para la conservación de recursos como: el agua, el suelo, la atmosfera; de la misma manera contribuyen con la protección de espacios vulnerables, evitando deslave a orillas de los ríos y en relieves inclinados, que con el deterioro de los bosques fuese imposible impedir.

Para ello, el Ministerio del Ambiente y los Recursos Naturales (2000), citado por Rojas (2007b), señala que: "mantener la cobertura boscosa y la figura de Reserva Forestal bajo una visión estratégica que permita develar los cambios y oportunidades del contexto global en función del desarrollo forestal comunitario, salvaguardando la identidad campesina..." (pág. 138).

Situación similar, se presenta en el Municipio Cruz Paredes, en el que se ubica el Parque Natural La Ciénaga, que es una zona de bosque húmedo tropical, con diferentes manantiales y caminerías a cargo del grupo ecológico de esta institución, el cual es una organización comunitaria para la conservación y preservación ambiental; sin embargo, en algunas comunidades tal como lo refleja reseñas históricas locales a través de pequeños escritos por los habitantes, los que indican que desde sus asentamientos en la década de los 60 , se alojaron en estas tierras por la variedad y riqueza en flora y fauna silvestre de las mismas.

De acuerdo a lo expuesto, en relación a la problemática que enfrenta el planeta, se instó a la realización de la investigación cuyo propósito fue la Reforestación como estrategia ambiental para la conservación de ríos y quebradas.

\subsection{Población y Muestra}

En la presente investigación, la población quedo conformada por 30 habitantes (18 hombres y 12 mujeres), en edades comprendidas entre 21 y 75 años respectivamente, de la comunidad Socó de la Parroquia Barrancas, municipio Cruz Paredes del estado Barinas. En consecuencia, es oportuno 
indicar que la población en estudio, es la cantidad de individuos, que forman parte de la investigación y que son necesarios para obtener la información, de acuerdo con Fidias (2012a), en términos más precisos, el autor afirma que la población: "es un conjunto finito o infinito de elementos con características comunes para los cuales serán extensivas las conclusiones de la investigación..." (pág. 81).

En tal sentido, Fidias (2012b), expresa que: "la muestra es un subconjunto representativo y finito que se extrae de la población accesible" (pág. 83); tomando como muestra el $100 \%$ de la población, por cuanto se conoce las unidades que la integran y es una población finita debido a que está conformada por un número inferior a cien unidades.

\subsection{Técnicas e Instrumentos para la Recolección de Datos}

La modalidad de la encuesta, es un cuestionario en el que se plasma de forma escrita la opinión de los encuestados; Fidias (2012c), la define: "como una técnica que pretende obtener información que suministra un grupo 0 muestra de sujetos acerca de sí mismos, o en relación con un tema en particular" (pág. 72). Por lo tanto, se especifica que el instrumento está compuesto por 18 ítems, con alternativas de respuesta: Siempre (S), Casi siempre (C.S), Algunas veces (A.V), Casi nunca (C.N) y Nunca (N); basándose en la escala de Likert que es la que mide la tendencia positiva o negativa de una actitud u opinión con la finalidad de que el investigador formule preguntas a las personas capaces de aportar datos de interés y de esta manera recoger toda la información necesaria para esta investigación.

\section{Resultados}

\subsection{Técnicas de Análisis de los Datos}

El análisis e interpretación de los datos de acuerdo con Balestrini (2006): se convierte en "una fase de la aplicación de la lógica deductiva e 
inductiva en el desarrollo de la investigación. Para esta estrategia los datos según sus partes se clasifican agrupándolos dividiéndolos y subdividiéndolos atendiendo a sus características y posibilidades..." (pág. 170). Una vez que se aplicó el instrumento y obtenida la información, se procedió a la tabulación de las respuestas ítems por ítems, luego se determinó la frecuencia simple y posteriormente se obtuvo los porcentajes. La información se vació en una tabla de doble entrada con el fin de obtener una visión global de los resultados. Para poder procesar la información se asignaron los siguientes valores a los ítems de acuerdo a la opinión de los encuestados: 5. Siempre; 4. Casi Siempre; 3. Algunas Veces; 2. Casi Nunca; y 1. Nunca. Esta tabulación contribuyo con la cuantificación de los datos la cual permitió presentar la información en una tabla y un gráfico.

Luego de obtenidos los datos se procedió al análisis e interpretación mediante el uso de técnicas estadísticas inferenciales, para ello se cruzó la información suministrada por los sujetos de investigación y se relacionó con las aplicaciones que permitieron dimensionar las incidencias de los hallazgos o resultados de la investigación.

Tabla 1. Planificación de proyectos ambientales con la integración de la familia y la sociedad.

\begin{tabular}{|c|c|c|c|c|c|c|c|c|c|c|c|}
\hline \multirow{2}{*}{ 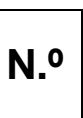 } & \multirow{2}{*}{ Planteamiento } & \multicolumn{2}{|c|}{ S } & \multicolumn{2}{|c|}{ CS } & \multicolumn{2}{|c|}{ AV } & \multicolumn{2}{|c|}{ CN } & \multicolumn{2}{|c|}{$\mathbf{N}$} \\
\hline & & $f$ & $\%$ & $f$ & $\%$ & $f$ & $\%$ & $f$ & $\%$ & $f$ & $\%$ \\
\hline 1 & $\begin{array}{c}\text { Se planifican en la } \\
\text { comunidad proyectos } \\
\text { ambientales que impulsen } \\
\text { la integración de la familia, } \\
\text { sociedad y el ambiente. }\end{array}$ & & & & & 12 & 40 & 18 & 60 & & \\
\hline
\end{tabular}

Fuente: El Autor (2017). 
Gráfico 1. Distribución porcentual de la planificación de proyectos ambientales con la integración de la familia y la sociedad.
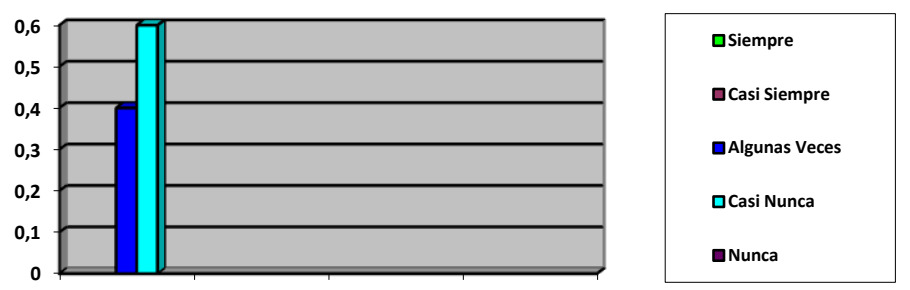

Fuente: El Autor (2017).

En la tabla 1 y el gráfico 1, el $40 \%$ de la población encuetada expresó que Algunas Veces se planifican en la comunidad proyectos ambientales de integración. Por otra parte, el $60 \%$ de la muestra manifestó que Casi Nunca se planifican en la comunidad proyectos ambientales que impulsen la integración de la familia, sociedad y el ambiente.

\section{Conclusiones}

Mediante el proyecto la reforestación como estrategia ambiental para la conservación de ríos y quebradas, se logró sensibilizar a los pobladores, y a su vez la participación de los mismos en la reforestación de la cuenca. De la misma manera, se orientó a la comunidad para el cuido y conservación de las especies forestales plantadas, así como las ya existentes.

Tomando en cuenta el primer componente de la educación ambiental referido a la necesidad básica de Fundamentos Ecológicos se desarrollaron actividades como charlas, dinámicas participativas sobre los recursos naturales y las áreas protegidas con el fin de profundizar y aumentar los conocimientos sobre el funcionamiento de la naturaleza, que es la base para transformar el nivel de sensibilización y concienciación humana.

Se estimuló a los habitantes de la comunidad a través de actividades 
como la reforestación de especies protectoras, a fin de contribuir con la conservación y mitigar el deterioro ambiental.

De acuerdo con los razonamientos que se han venido desarrollando y pro de dar respuesta a los objetivos propuestos; con la ejecución de investigación se instruyó a la comunidad en general a través de un Taller de Sensibilización sobre la Importancia de la Reforestación, en el caso particular la siembra de Bambú, en donde se mostró a los presentes diversas diapositivas alusivas a bosques reforestados, acompañadas de Charlas sobre los importantes beneficios que proporciona la reforestación en la comunidad y el ambiente en general.

De la misma manera, y a través de un Taller para dar a conocer las estrategias aplicables en la reforestación, se orientó al Consejo Comunal en función de la siembra de árboles (bambú), con el propósito de dar respuesta a la necesidad de reforestación, en donde los pobladores partiendo de un conversatorio expusieron los puntos de vista y la preocupación en cuanto a la desidia de los habitantes, relacionada con deforestación del lugar y el poco interés por la conservación del ambiente, haciendo énfasis en la necesidad de la reforestación a fin de proporcionar a la población un ambiente acorde a las necesidades mínimas para la subsistencia de la vida en el planeta.

En tal sentido, en consonancia a las definiciones de estrategias y a través de Jornadas de reforestación, se realizó la siembra de doscientos cincuenta (250) plantas de bambú "Guadua angustifolia Kunth", aplicando estrategias para conservación de la biodiversidad, ya que los bosques tienen una proporción significativa para el mundo y que la pérdida de estos constituye una causa que conduce al detrimento de especies; se dio a conocer y a su vez se comprometió al consejo comunal de la comunidad al cuido de las plantaciones posterior a la siembra, considerando que es importante a fin de evitar el daño de especies por diferentes factores (deterioro de animales, quema, malezas, entre otros) que pudieran atentar contra el buen desarrollo 
de las plantaciones del lugar.

\section{Referencias}

Balestrini, M. (2006). Cómo se elabora el proyecto de investigación. 7ma. Edición, ISBN: 980-6293-03-7. Caracas, Venezuela: BL Consultores Asociados, Servicio Editorial.

Fidias, A. (2012a,b,c). El Proyecto de investigación. Introducción a la metodología científica. 6ta. Edición, ISBN: 980-07-8529-9. Caracas, Venezuela: Editorial Espíteme, C.A. Recuperado de: https://ebevidencia.com/wp-content/uploads/2014/12/EL-PROYECTODE-INVESTIGACI\%C3\%93N-6ta-Ed.-FIDIAS-G.-ARIAS.pdf

González, D. (2016). Proyecto de reforestación de la quebrada La Pilama, mediante la implementación de dos viveros para el abastecimiento de material vegetal en la vereda Liberia (Viotá Cundinamarca). Fusagasugá, Colombia: Facultad de Ciencias Agropecuarias de la Universidad de Cundinamarca.

Hernández, E. (2011). Gestión del patrimonio natural. Modulo 6. Programa de Desarrollo de Capacidades para el Caribe. Para el Patrimonio Mundial. La Habana, Cuba: UNESCO - Exclusivas Latinoamericanas ELA, S.L. Recuperado de: http://whc.unesco.org/document/107154 Hernández, J. (2010). Prácticas de Reforestación. Manual Básico. Zapopan, Jalisco, México: Conafor - Semarnat. Recuperado de: http://www.ccmss.org.mx/acervo/practicas-de-reforestacion-manualbasico/

Lara, D. (2017). Determinación de cambios de distribución de especies por efectos del cambio climático en el parque municipal natural Campo Alegre y parque regional natural Ucumarí en Risaralda Colombia. Tesis. Bogotá, Colombia: Universidad Distrital Francisco José de Caldas. Recuperado de: 
http://repository.udistrital.edu.co/bitstream/11349/5403/1/LaraBallester osDiana2017.pdf

Mendoza, A. (2017). Reforestación con el Mijao para el Fortalecimiento de

la Biodiversidad. Revista Scientific, 2(4), 11-25, e-ISSN: 2542-2987.

Recuperado de:

https://doi.org/10.29394/scientific.issn.2542-2987.2017.2.4.1.11-25

Rodríguez, L. (s.f.). La estrategia medioambiental y la formación del profesional. [Documento en línea]. Holguín, Cuba: Universidad de Holguín "Oscar Lucero Moya". Recuperado de:

https://www.monografias.com/trabajos75/estrategia-medioambientalformacion-profesional/estrategia-medioambiental-formacionprofesional2.shtml

Rojas, J. (2007a,b). Regulación ambiental y colonización agraria en reservas de bosque. El drama de Ticoporo, estado BarinasVenezuela. Revista Geográfica Venezolana, 48(1), 129-141, e-ISSN: 1012-1617. Recuperado de:

http://www.saber.ula.ve/handle/123456789/24694

UPEL (2016a,b). Manual de Trabajos de Grado de Especialización y Maestría y Tesis Doctorales. 5ta Edición, ISBN: 980-273-441-1. Caracas, Venezuela: Fondo Editorial de la Universidad Pedagógica Experimental Libertador, FEDUPEL. 


\section{Molina Pereira Yely Alexander \\ e-mail: molinaalex1605@gmail.com}

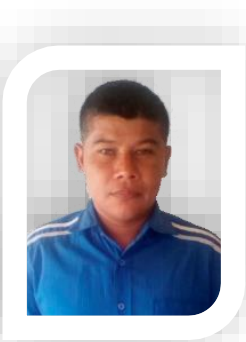

Nacido en el estado Táchira, Venezuela, el 8 de enero del año 1984. Lcdo. en Educación, mención Matemática y Desarrollo cultural; En el año 2006, comencé a laborar el en Ministerio del Poder Popular para la Educación; Soy Docente de Aula del Núcleo Escolar Rural 317 y el Liceo Nacional "Los Mangos"; en el Liceo Nacional José Félix Ribas, cumplí funciones de coordinador de Seccional, desde el año 2004 al año 2014; también laboré en la Fundación Misión Ribas, desempeñando la función de Facilitador y posteriormente Coordinador de Ruta. Actualmente me desempeño como docente de Apoyo del Centro Local de Investigación y Formación Permanente del Magisterio Venezolano "Profesor Julio Chávez" (CLIFPM), del municipio Cruz Paredes.

El contenido de este manuscrito se difunde bajo una Licencia de Creative Commons ReconocimientoNoComercial-Compartirlgual 4.0 Internacional 